\title{
An approach for modelling quasi-stationary magnetic circuits
}

\author{
Nick Raabe \\ Sterling Industry Consult $\mathrm{GmbH}$ \\ Lindenstraße 170, 25524 Itzehoe, Germany \\ nick.raabe@sterlingsihi.de
}

\begin{abstract}
For the design of electrical machines the magnetic circuit has to be modeled. If only the winding layout or the stack length of the motor is changed a complete FEA analysis mostly is not necessary. In this case Modelica is well suited to model the magnetic circuit for quasi-stationary simulations. A new library based on existing standard libraries MagneticQS is presented. An induction motor example under no-load conditions shows the basic concept of this library. To enhance and improve the library new models for different types of machines and the possibility of an integral simulation independent from the load conditions is planned.

Keywords: electrical machines; magnetic library; quasi-stationary magnetic circuits
\end{abstract}

\section{Introduction}

Up to now the Modelica Standard Library (MSL) contains two packages with different magnetic connectors. Both are subpackages of Modelica.Magnetic: FluxTubes [1] and FundamentalWave [7]. The Modelica concept of providing one potential and one flow variable is implemented here by using the magnetic voltage $V_{\mathrm{m}}(\mathrm{A})$ and the magnetic flux $\Phi(\mathrm{Vs})$. The variables in the FluxTube-package are of type Real. The change of the magnetic flux with respect to time leads to an induced voltage. This package is suitable for all types of transient induction problems. The FundamentalWave-package provides the same variables but they are of type Complex. These connectors are used for modelling multiphase electric machines in transient operation. The machines presented in this package are identical from the outside behaviour to the machines in Modelica.Electrical.Machines. The user has the choice between transforming the electrical stator quantities to space phasors (Modelica.Electrical. Machines) or to the magnetic circuit (Modelica.Ma-
gnetic.FundamentalWave). The equivalence of both models is shown in Modelica.Magnetic.FundamentalWave.Examples.BasicMachines.AIMC_DOL.

\section{Why another magnetic library?}

When designing electric machines the first step is to find a proper geometry. This means to find the best shape for stator and rotor slots, the diameter of the machine and the stack length. Once the winding layout is defined the magnetic circuit can be calculated to determine the magnetizing curve of the machine. This iterative design process means either using a FEA tool or analytical algorithms. After defining the geometry of the machine there are still many options to deviate from this in day-to-day business, e.g. the winding layout can be changed or the quality of the laminations. In this case the FEA mostly is not suitable due to its complexity. This is why Modelica is very helpful to implement a magnetic circuit that is based on algorithms known from the literature but a lot more flexible and clearly described.

These quasi-stationary problems can hardly be simulated with the two existing magnetic packages. Since MSL 3.2 there is the Modelica.Electrical.QuasiStationary package available which unfortunately has no connection to the magnetic domain yet. The goal of this paper is to introduce a new magnetic library MagneticQS which is similar to the existing ones but takes into account some special requirements for the design of electric machines.

\section{Basic concept}

The connectors of MagneticQS contain complex variables so that they are equal to the connectors of FundamentalWave. The difference is that the law of in induction is also defined in a complex way. Instead of 
saying $v_{\text {ind }} \sim \mathrm{d} \Phi / \mathrm{d} t$ the quasi-stationary representation $v_{\text {ind }} \sim j \omega \Phi$ is used. The transformation between electric and magnetic domain is done by the ElectroMagneticConverter. Listings 1 and 2 show the difference of this converter taken from FluxTubes and MagneticQS.

Listing 1: FluxTube converter

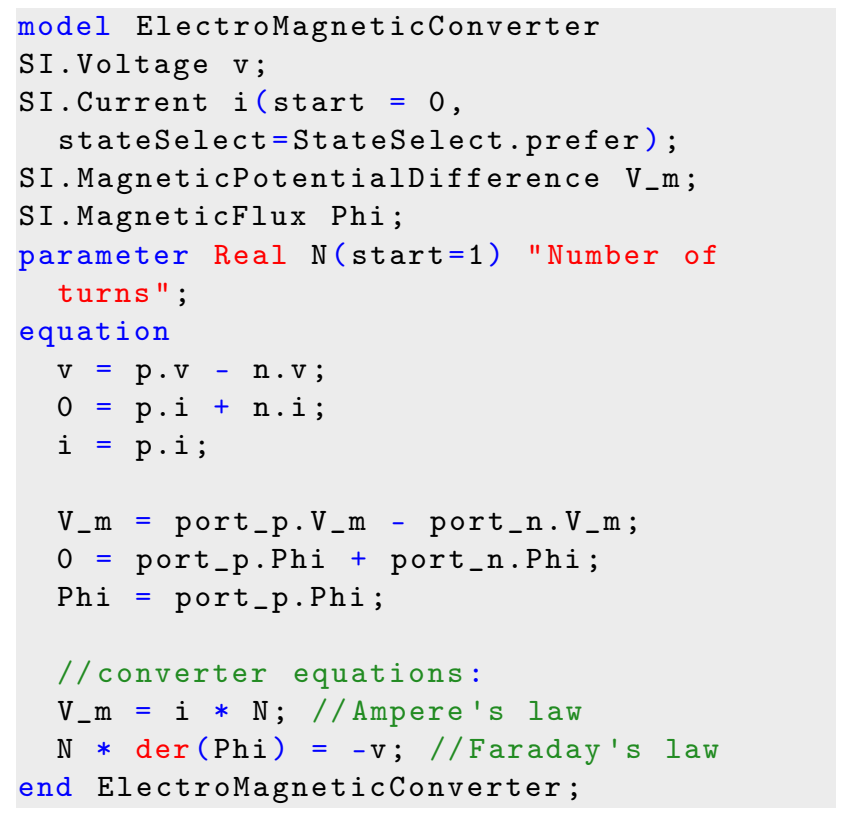

Listing 2: MagneticQS converter

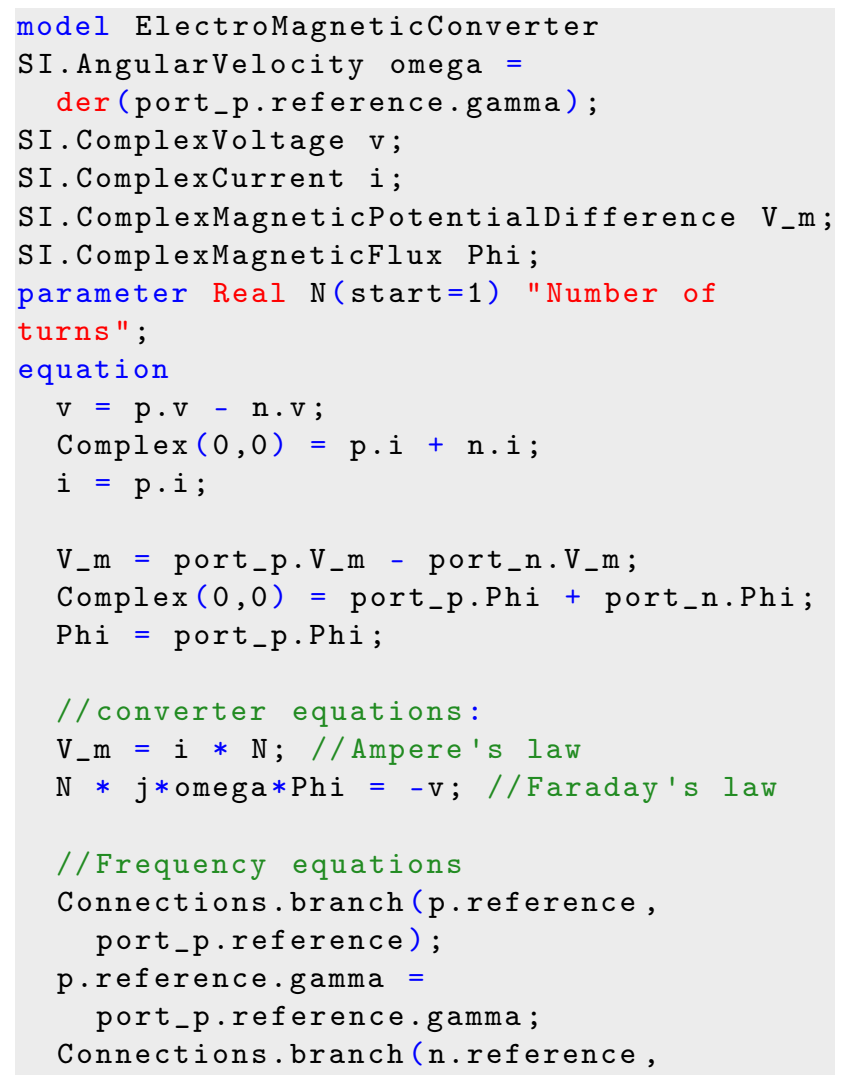

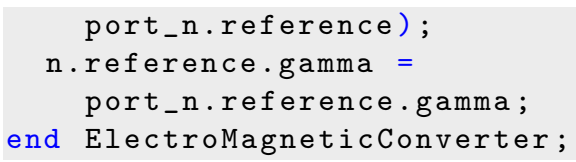

The main equations are the same for both libraries. The only change is that MagneticQS contains complex variables. The specific characteristic of the Modelica. Electric.QuasiStationary has to be taken into account: The frequency needs also to be considered and transported from one domain to another.

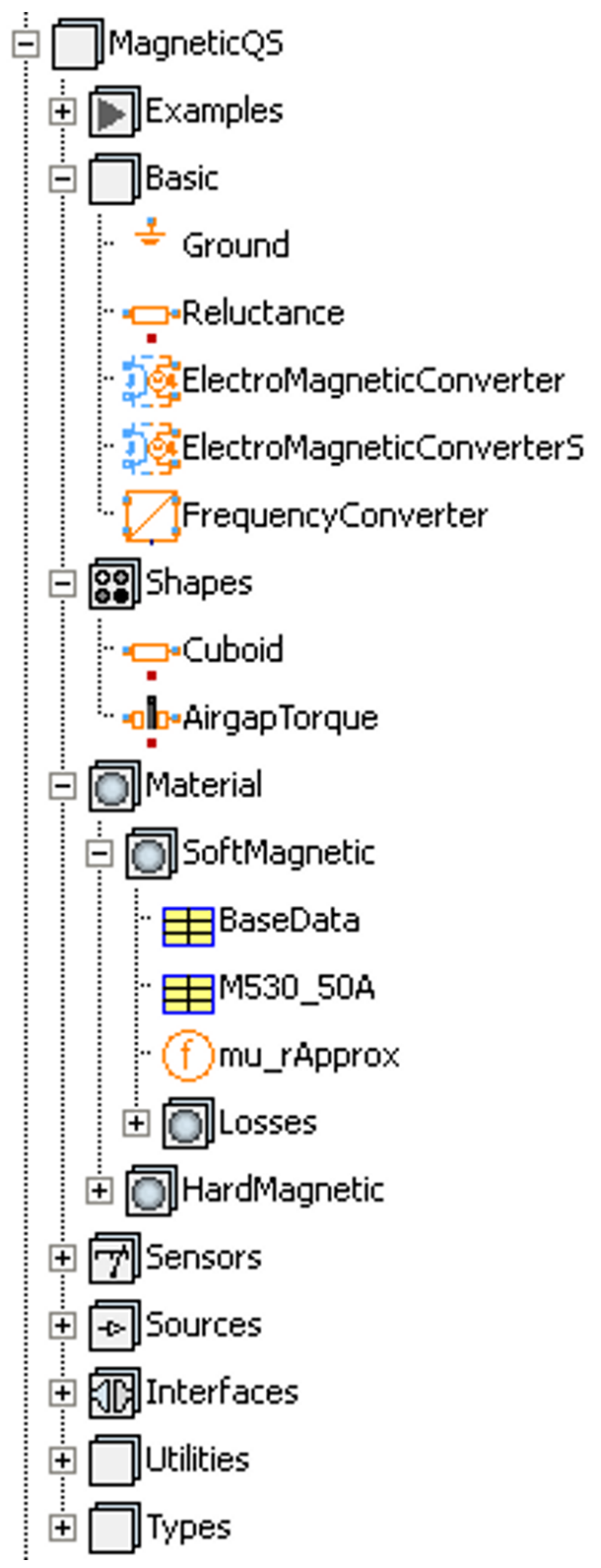

Figure 1: MagneticQS library layout

Once again the differences of the three magnetic libraries (see section 2) should be clarified in the following listing:

- FluxTubes

- Flux and magnetic voltage are of type Real 
- Derivative of flux used in Faraday's law

- Link to electrical domain via Modelica. Electric.Analog. Basic

- Best choice for transient magnetics (e.g. moving actuators)

- FundamentalWave

- Flux and magnetic voltage are of type Complex

- Derivative of flux used in Faraday's law

- Link to electrical domain via Modelica. Electrical.Machines.SpacePhasors

- Best choice for space-phasor magnetics (e. g. transient operation of electrical machines)

- MagneticQS

- Flux and magnetic voltage are of type Complex

- Angular frequency used in Faraday's law (no derivative of flux)

- Link to electrical domain via Modelica. Electric.QuasiStationary

- Best choice for quasi-stationary magnetics (e. g. magnetic circuits in electrical machines)

\section{Complex permeability}

As long as ideal reluctances are considered the frequency has no impact on the magnetic flux and potential. The magnetic circuit acts as a coil (in air) and consumes reactive power. For the calculation of iron losses the frequency and the geometry of the flux path haven to be taken in to account. If losses are present the magnetic two-pole not only consumes reactive power but also produces heat (active power). Therefore a heat port is added like it is known from an ohmic resistance in the MSL.

The iron losses consist of two parts: hysteresis and eddy current losses. It is quite common to define the iron losses as the sum of both parts depending on the square of the flux density [5]:

$$
P_{\mathrm{fe}}=k_{\mathrm{fe}} \cdot P_{15} \cdot\left(\frac{f}{50 \mathrm{~Hz}}\right)^{k_{\mathrm{freq}}} \cdot\left(\frac{B}{1 \mathrm{~T}}\right)^{2} m_{\mathrm{fe}} .
$$

$k_{\mathrm{fe}}$ is a correction coefficient that depends on the type of machine (synchronous, asynchronous, DC) and the part of the magnetic circuit (tooth or yoke). $P_{15}$ is listed in standards (e. g. IEC 60404-8-4 [2]) and is also given in material certificates by the lamination
Table 1: Examples for laminations according to IEC 60404-8-4 [2]

\begin{tabular}{lrrr}
\hline Lamination & $\begin{array}{r}\text { Thickness } \\
\mathrm{mm}\end{array}$ & $\begin{array}{r}P_{15} \\
\mathrm{~W} / \mathrm{kg}\end{array}$ & $\begin{array}{r}P_{10} \\
\mathrm{~W} / \mathrm{kg}\end{array}$ \\
\hline M270-50A & 0.50 & 2.7 & 1.1 \\
M400-50A & 0.50 & 4.0 & 1.7 \\
M800-65A & 0.65 & 8.0 & 3.6 \\
\hline
\end{tabular}

manufacturer. This value specifies the losses per $\mathrm{kg}$ in $\mathrm{W}$ and is determined for $50 \mathrm{~Hz}$ and $1.5 \mathrm{~T}$. As an alternative also $P_{10}(50 \mathrm{~Hz}, 1 \mathrm{~T})$ can be used. Typical values are given in table 1 . If the frequency differs from $50 \mathrm{~Hz}$ the exponent $k_{\text {freq }}$ (determined emperically) corrects the losses.

In the quasi-stationary domain it is also possible to define the relative permeability as a complex number. Hence the real part describes the magnetic behaviour and the imaginary part describes the losses [3]:

$$
\underline{\mu}_{\mathrm{r}}=\mu^{\prime}-j \mu^{\prime \prime}
$$

Consequently the reluctance becomes a complex magnetic impedance:

$$
\underline{Z}_{\mathrm{m}}=R_{\mathrm{m}}+j \omega L_{\mathrm{m}}
$$

For a cuboid it can be calculated from the geometry ( $l$ : length, $A$ : cross section):

$$
\underline{Z}_{\mathrm{m}}=\frac{l}{\mu_{0} \underline{\mu}_{\mathrm{r}} A} .
$$

Thus for the magnetic resistance and the magnetic inductance one can write:

$$
\begin{aligned}
& R_{\mathrm{m}}=\frac{l}{\mu_{0} A} \cdot \frac{\mu^{\prime}}{\mu_{\mathrm{r}}^{2}}, \\
& L_{\mathrm{m}}=\frac{l}{\mu_{0} A} \cdot \frac{\mu^{\prime \prime}}{\mu_{\mathrm{r}}^{2}} .
\end{aligned}
$$

In analogy to electric circuits the effects of resistance and inductance change: The magnetic resistance $R_{\mathrm{m}}$ leads to reactive power (corresponds with $\mu^{\prime}$ ), whereas the magnetic inductance $L_{\mathrm{m}}$ produces losses (corresponds with $\mu^{\prime \prime}$ ).

$\mu^{\prime}$ is defined by the approximation function for the magnetizing curve explained in the FluxTubes-library. In most cases $L_{\mathrm{m}}$ and $\mu^{\prime \prime}$ are unknown but $P_{\mathrm{fe}}$ is known so that an additional equation based on the power balance is needed to calculate them: 
Table 2: Data of example motor [4]

\begin{tabular}{llr}
\hline Nominal power & $P_{\mathrm{n}}$ & $11 \mathrm{~kW}$ \\
Nominal voltage & $U_{\mathrm{n}}$ & $380 \mathrm{~V}$ \\
Nominal frequency & $f_{\mathrm{n}}$ & $50 \mathrm{~Hz}$ \\
No. polpairs & $p$ & 2 \\
No. stator slots & $Z_{1}$ & 36 \\
No. rotor slots & $Z_{2}$ & 28 \\
Stator winding factor & $\xi_{1}$ & 0.945 \\
No. turns per phase & $w_{1}$ & 168 \\
Stack length & $l_{\text {stack }}$ & $160 \mathrm{~mm}$ \\
\hline
\end{tabular}

$$
L_{\mathrm{m}}=\frac{P_{\mathrm{fe}}}{\omega^{2} \cdot \Phi^{2}} .
$$

These equations are part of the model MagneticQS. Shapes. Cuboid. Up to now only one type of shape is implemented: the cuboid. Since every part of electrical machines (e.g. yoke, tooth) is simplified when modelling magnetic circuits to a rectangular shape this is not a limitation at this early stage of the library. However for further developements other shapes might be useful.

\section{Example: Induction machine un- der no-load condition}

In order to verify the proposed implementation an induction machine is modeled under no-load condition. The motor design is taken from [4]. Table 2 shows the nominal data of the motor, the geometry is given in figure 2 .

According to the calculation in [4] the magnetic circuit is divided into five parts:

- stator yoke,

- stator teeth,

- airgap,

- rotor teeth,

- rotor yoke.

For analytical calculations (in contrast to FEA) some special restrictions and simplifications apply:

- The field strength in the stator yoke is strongly nonlinear. Therefore either an additional magnetizing curve for this part of the magnetic circuit is given or a reduction factor [5].

- The flux density in the airgap depends on the width of the stator and rotor slot openings. The airgap length is increased by the so called CarterFactor.

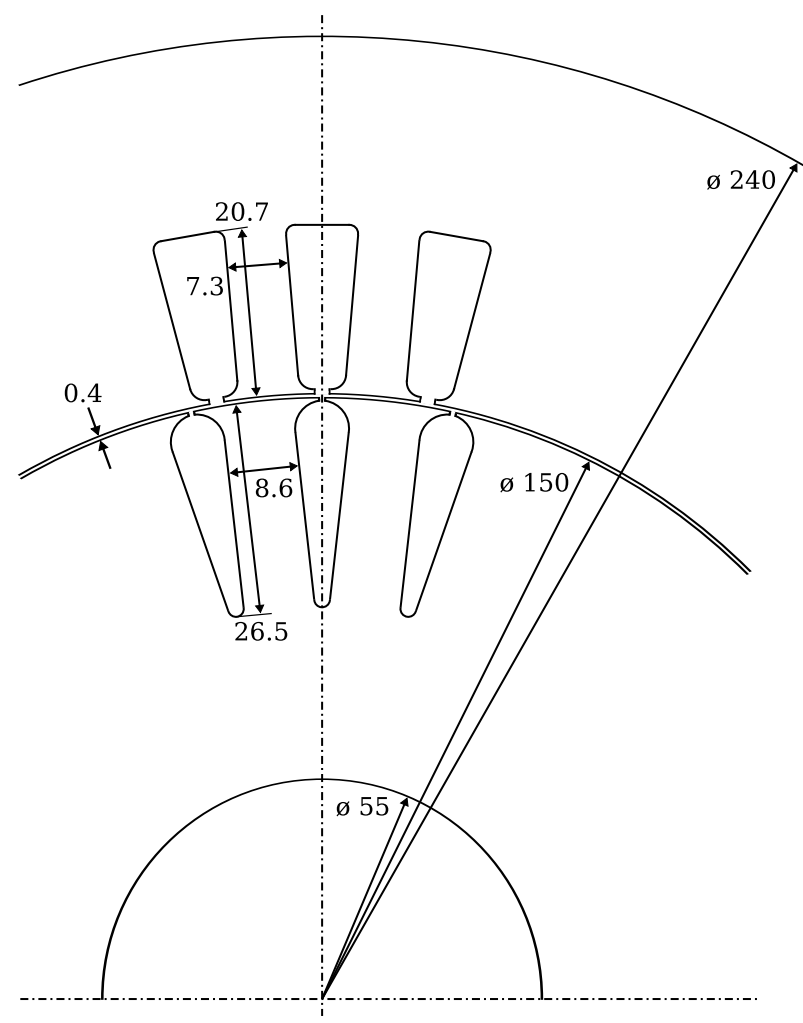

Figure 2: Stator and rotor geometry [4]

- The magnetic behaviour of the rotor shaft is handled by defining one third of the diameter as iron [4] so that the rotor yoke is enlarged.

- Stray inductances are not taken into account. This approximation is only valid under no-load condition.

The MagneticQS-representation is shown in figure 3. Each magnetic impedance is a MagneticQS. Shapes. Cuboid. In accordance to [4] only stator yoke and tooth produce losses which is feasible due to the very low frequency in the rotor. In comparison with the calculations in [4] the following deviation has to be mentioned: The book neglects the stator resistance which is quite common when calculating magnetic circuits by hand.

The connection of the electrical and magnetic domain is performed by the model MagneticQS.Basic. ElectroMagneticConverterS which is adapted to rotating electrical machine but still based on the converter presented in section 2 :

$$
\begin{aligned}
\underline{V}_{\mathrm{m}} & =j \cdot \underline{I}_{1} \cdot \frac{3 \sqrt{2} \cdot \xi_{1} \cdot w_{1}}{p \cdot \pi}, \\
-\underline{U}_{1} & =\omega \underline{\Phi} \cdot \frac{\xi_{1} \cdot w_{1}}{\sqrt{2}} .
\end{aligned}
$$

Table 3 shows the simulation results for the magnetiz- 


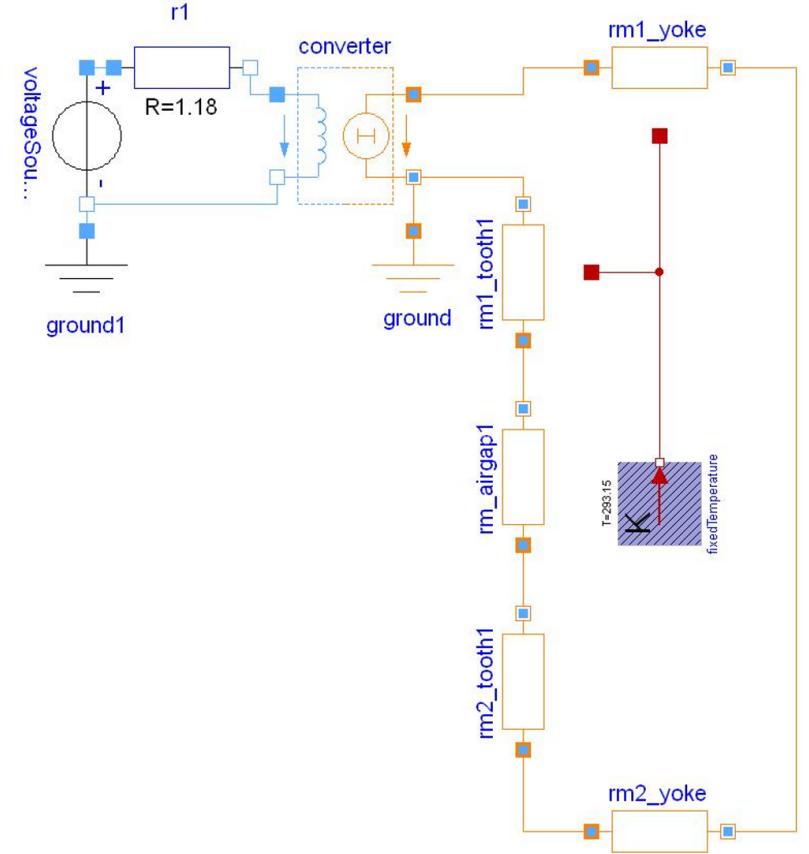

Figure 3: Induction machine no-load example

Table 3: Simulation results (Indices: y: yoke, t: tooth, cu: stator resistance)

\begin{tabular}{lrrrr}
\hline Calculation & $I_{1}$ & $P_{\mathrm{cu}}$ & $\begin{array}{r}P_{\mathrm{y}} \\
\mathrm{A}\end{array}$ & $\begin{array}{r}P_{\mathrm{t}} \\
\mathrm{kW}\end{array}$ \\
\hline by hand [4] & 5.19 & 0.00 & 192.05 & 85.55 \\
MagneticQS & 5.20 & 31.87 & 190.97 & 85.14 \\
MagneticQS, $R_{1}=0$ & 5.23 & 0.00 & 191.88 & 85.39 \\
\hline
\end{tabular}

ing current and the losses.

When neglecting all losses the example shows the same results as in [4]. By introducing losses in MagneticQS slight deviations (see second row of table 3) become present. This proofes that the simplifications for hand calculations are valid. The minor influence of the stator resistance is shown in the third row of table 3.

The results show that MagneticQS is well suited for the magnetic circuit implementation of electric machines. In comparision with analytical hand calculations it is e.g. no more necessary to calculate the magnetic behaviour and the losses in two steps. MagneticQS delivers an integral solution for magnetic circuits in quasi-stationary mode.

\section{Summary and future work}

This article presents a new magnetic library called MagneticQS. It is based on existing libraries but intro- duces complex variables. The purpose is a clear physical modelling of quasi-stationary magnetic circuits. These are needed in the design phase of electrical machines. The library is designed similar to the existing ones in the MSL. To fulfil the requirements on physical modelling a complex permeability is also introduced. The simulation results show that the new library is well suited to assist the design process for electrical machines. The next step for developing the library is to test different types of machines under load conditions and compare the results with analytical algorithms and FEA. Once this goal is achieved an integral electrical machine magnetic circuit model can be implemented that can be used independently from the state of the machine (no-load, load) which is a great advantage in comparison with existing analytical models. Additional research is needed to find general approaches that eliminate the restriction mentioned in section 5 .

\section{References}

[1] Bödrich T. Electromagnetic Actuator Modelling with the Extended Modelica Magnetic Library. Modelica 2008 Conference, Bielefeld, Germany, pp. 221-227, March 3-4, 2008.

[2] IEC 600404-8-4 Magnetic materials - Part 8-4: Specifications for individual materials - Coldrolled non-oriented electrical steel sheet and strip delivered in the fully-processed state. 1998.

[3] Coey J-M-D. Magnetism and Magnetic Materials. 2009.

[4] Vaske P, Riggert J-H. Elektrische Maschinen und Umformer Teil 2: Berechnung elektrischer Maschinen (Calculation of electrical machines). 1974.

[5] Pyrhönen J, Jokinen T, Hrabovcova V. Design of Rotating Electrical Machines. John Wiley \& Sons. 2008.

[6] Richter R. Elektrische Maschinen Band 1 (Electrical machines part 1). 3rd edition. Birkhäuser Verlag. 1967.

[7] Kral C, Haumer A. The New FundamentalWave Library for Modeling Rotating Electrical Three Phase Machines. Modelica 2011 Conference, Dresden, Germany, March 20-22, 2011. 
